\title{
Noninvasive Brain Stimulation Enhances Memory Acquisition and Is Associated with Synaptoneurosome Modification in the Rat Hippocampus
}

\author{
Seung Ho Jung, ${ }^{1,2,3}$ C Candice Hatcher-Solis, ${ }^{1}$ Raquel Moore, ${ }^{1,4}$ Naomi Bechmann, ${ }^{1,4}$ Sean Harshman, ${ }^{5,6}$ \\ Jennifer Martin, ${ }^{6}$ and Ryan Jankord ${ }^{1}$
}

https://doi.org/10.1523/ENEURO.0311-19.2019

${ }^{1}$ Applied Neuroscience Branch, 711th Human Performance Wing, Air Force Research Laboratory, Wright-Patterson AFB, OH 45433, ${ }^{2}$ Research Associateship Program, National Research Council, National Academies of Science, Washington, DC 200001, ${ }^{3}$ ORISE, Oak Ridge, TN 37830, ${ }^{4}$ Infoscitex, Dayton, OH 45431, ${ }^{5}$ UES, Inc, Dayton, OH 45432, and ${ }^{6}$ Human Signatures Branch, 711th Human Performance Wing, Air Force Research Laboratory, WrightPatterson AFB, $\mathrm{OH} 45433$

\begin{abstract}
Transcranial direct-current stimulation (tDCS) is a non-invasive brain stimulation approach previously shown to enhance memory acquisition, but more studies are needed to elucidate the underlying mechanisms. Here, we examined the effects of anodal tDCS $(0.25 \mathrm{~mA}$ for $30 \mathrm{~min})$ on the memory performance of male Sprague Dawley rats in the passive avoidance test (PAT) and the associated modifications to the hippocampal proteomes. Results indicate anodal tDCS applied before the acquisition period significantly enhanced memory performance in the PAT. Following PAT, synaptoneurosomes were biochemically purified from the hippocampi of tDCS-treated or sham-treated rats and individual protein abundances were determined by bottom-up liquid chromatography mass spectrometry analysis. Proteomic analysis identified 184 differentially expressed hippocampal proteins when comparing the sham to the tDCS before memory acquisition treatment group. Ingenuity pathway analysis (IPA) showed anodal tDCS before memory acquisition significantly enhanced pathways associated with memory, cognition, learning, transmission, neuritogenesis, and long-term potentiation (LTP). IPA identified significant upstream regulators including bdnf, shank3, and gsk3b. Protein-protein interaction (PPI) and protein sequence similarity (PSS) networks show that glutamate receptor pathways, ion channel activity, memory, learning, cognition, and long-term memory were significantly associated with anodal tDCS. Centrality measures from both networks identified key proteins including dlg, shank, grin, and gria that were significantly modified by tDCS applied before the acquisition period. Together, our results provide descriptive molecular evidence that anodal tDCS enhances memory performance in the PAT by modifying hippocampal synaptic plasticity related proteins.
\end{abstract}

\section{Significance Statement}

We investigated whether anodal transcranial direct-current stimulation (tDCS) affects memory performance and the underlying protein modifications in hippocampal synaptoneurosomes. We found that anodal tDCS administered before memory acquisition significantly enhanced memory performance by enhancing the expression of hippocampal proteins associated with glutamate signaling and ion channel activity. Our results identify molecular targets for tDCS-induced memory enhancement and the associated signaling pathways. Our behavioral and proteomics study further elucidates the mechanism for tDCS effects on acquisition memory and may lead to the development of therapeutics to enhance memory and learning process to treat for neurologic diseases and psychological disorders. 
Key words: hippocampal proteomics; hippocampal synaptoneurosome; memory process; protein sequence similarity network; protein-protein interaction network; transcranial direct current stimulation

\section{Introduction}

Transcranial direct-current stimulation (tDCS) is widely used clinically due to its non-invasive application and few reported side effects (Stagg and Nitsche, 2011; Holmes et al., 2016). Clinical studies have revealed the beneficial effects of tDCS as a therapeutic tool for neurologic diseases and psychological disorders including Alzheimer's disease, schizophrenia, depression, and anxiety (Marshall et al., 2004; Boggio et al., 2006; Göder et al., 2013; Ladenbauer et al., 2017; Hill et al., 2019; Medvedeva et al., 2019). Although the biological and molecular mechanisms remain unclear, tDCS has been shown to enhance memory. tDCS improves working memory (Hoy et al., 2013; Martin et al., 2014; Bogdanov and Schwabe, 2016), long-term memory (Javadi and Cheng, 2013), semantic memory (Cattaneo et al., 2011; Holland et al., 2011), and memory acquisition (Jacobson et al., 2012; Javadi and Walsh, 2012). Moreover, a study using elderly subjects showed that anodal tDCS significantly enhanced memory recall one week after learning compared to sham stimulation, suggesting long lasting effects of tDCS (Flöel et al., 2012). Although studies have shown that tDCS enhances memory, especially memory acquisition, there is limited biological data available for the underlying effects of tDCS on the hippocampal proteome, which may explain how tDCS induces memory enhancement.

Several studies in humans and animals have examined the effects of tDCS on neuronal activity and synaptic plasticity to understand the regulatory mechanisms. Electrophysiological studies have revealed that tDCS increases cortical excitability in humans (Alonzo et al., 2012; Romero Lauro et al., 2014; Bailey et al., 2016). Animal studies have shown that tDCS enhances synaptic plasticity in the hippocampus of rodents (Rohan et al., 2015; Podda et al., 2016). However, the molecular mechanisms of tDCS to enhance memory and learning are less known. tDCS has been shown to affect the expression of immediate early genes c-fos and zif268 in the hippocampus (Ranieri et al., 2012). Studies have further determined that tDCS affects the mRNA level of hippocampal brain-

Received August 6, 2019; accepted October 31, 2019; First published November 7, 2019.

The authors declare no competing financial interests.

Author contributions: S.H.J. and R.J. designed research; S.H.J., R.M., N.B., S.H., and J.M. performed research; S.H.J., C.H.-S., and J.M. analyzed data; S.H.J. and C.H.-S. wrote the paper.

This work was supported by Air Force Office of Scientific Research Grants 13RH14COR and 16RHCOR362 and has been approved for public release (Distribution A: Approved for public release, 88ABW Cleared July 5, 2019; 88ABW-2019-3239).

Acknowledgements: We thank Sylvia Cunningham and the Wright-Patterson Air Force Base Research Support Command for their contribution to this work.

Correspondence should be addressed to Seung $\mathrm{Ho}$ Jung at seung.jung.ctr@us.af.mil or seung.jung@gmail.com.

https://doi.org/10.1523/ENEURO.0311-19.2019

Copyright (C) 2019 Jung et al.

This is an open-access article distributed under the terms of the Creative Commons Attribution 4.0 International license, which permits unrestricted use, distribution and reproduction in any medium provided that the original work is properly attributed. derived neurotrophic factor (BDNF), a growth factor important for long-term memory (Podda et al., 2016; Kim et al., 2017). Additional studies indicate tDCS may regulate neurotransmitter signaling of glutamatergic, GABAergic and cholinergic pathways (Rossini et al., 2015; Giordano et al., 2017) that favor enhancement of memory and cognition. tDCS has also been shown to modify the expression of genes related to serotonergic, adrenergic, dopaminergic, GABAergic, and glutamatergic signaling in the rat cortical transcriptome (Holmes et al., 2016). A more recent study showed that tDCS modifies AMPA receptor phosphorylation and translocation in the rat hippocampus (Stafford et al., 2018), suggesting a possible effect of tDCS on protein modifications in rat hippocampal synaptoneurosomes. Another study reports that anodal tDCS enhances performance in the hippocampaldependent passive avoidance memory task and that the tDCS-induced enhancement was abrogated with pretreatment of ANA-12, an inhibitor of the BDNF receptor tropomyosin receptor kinase B (Yu et al., 2019). More studies are needed to associate tDCS-induced effects on hippocampal protein regulation with behavioral performance to determine mechanism.

In this study, we examined whether anodal tDCS affected memory acquisition and/or recall along with the molecular modifications of stimulation on synaptic proteomics in the rat hippocampus. We show that anodal tDCS (250 $\mu \mathrm{A}$ for $30 \mathrm{~min})$ applied before the memory acquisition period of a learning and memory test enhances cognitive performance. We report that the enhancement of memory acquisition by anodal tDCS is significantly related to molecular alterations in the hippocampal proteome. The tDCS-induced modifications in hippocampal synaptoneurosomes are significantly associated with receptor signaling and voltage-gated ion channel activity in pathways associated with learning, memory, and cognitive enhancement.

\section{Materials and Methods}

\section{Animals}

Adult Sprague Dawley rats (male, seven to eight weeks old weighing $\sim 400-500 \mathrm{~g}, n=14 /$ group) were purchased from Charles River Laboratories. Rats were housed in the animal facility of the Wright-Patterson Air Force Base (WPAFB) with ad libitum access to food and water and maintained on a 12/12 $\mathrm{h}$ light/dark cycle. Rats received a 10-d acclimation period before surgical electrode placement. All rats were maintained according to National Institutes of Health and WPAFB Institutional Animal Care and Use Committee guidelines. The study protocol was reviewed and approved in compliance with the Animal Welfare Act and with all applicable federal regulations governing the protection of animals in research.

\section{Surgical implantation of cranial electrode}

Animals were anesthetized with isoflurane (Med-Vet International) using $5 \%$ induction, followed by $2-3 \%$ iso- 


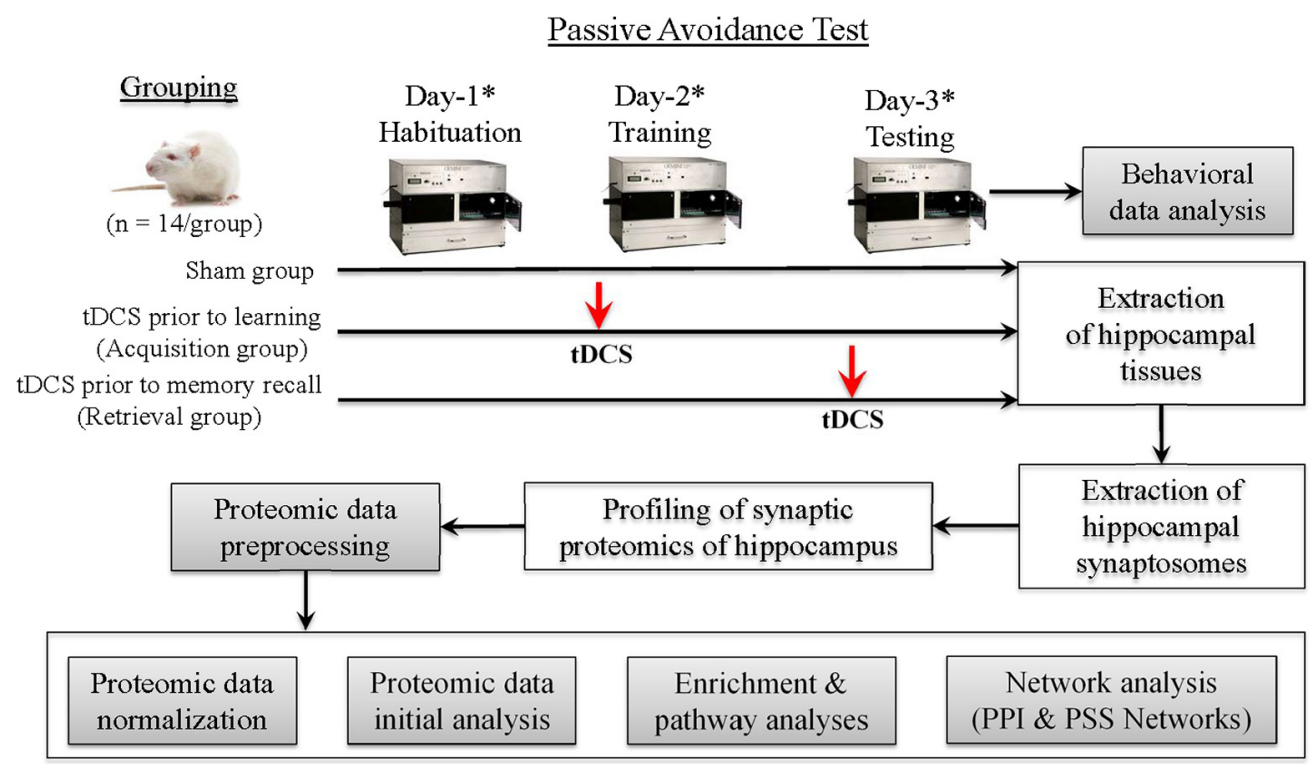

Figure 1. Overall research design. *Before rodents were exposed to the passive avoidance memory task, they were freely exposed to open field for $5 \mathrm{~min}$ (the acquisition day) and $3 \mathrm{~min}$ (the training and testing days) for exploration with familiar and novel objects similar to the novel object recognition task. Proteomic abundance data were first analyzed for the replicability within each group (Extended Data Fig. 1-1), and the abundance of 16 internal control proteins was compared between the groups (Extended Data Fig. 1-2). Proteomic data analyzed for this manuscript were provided as an Excel file (Extended Data Fig. 1-3).

flurane to maintain anesthetic depth. A 5-mm diameter, circular, head electrode casing (Tangible Solutions) was attached to the skull from 0 to $-5 \mathrm{~mm}$ bregma. Luting dental cement (GC Fuji I, GC America Inc.) was applied to the base of the head electrode casing and to the skull, followed by an acrylic dental cement (Sigma-Aldrich) to secure the electrode casing. Animals were given a minimum of $7 \mathrm{~d}$ as a recovery period before tDCS treatment. Rats were randomly selected for sham, anodal tDCS before acquisition, or anodal tDCS before memory recall.

\section{tDCS application}

On the same day, before stimulation, animals were acclimated to the testing room for $10 \mathrm{~min}$. A conducting medium (SignaGel, Parker Laboratories) was placed into the head casing before connecting the head electrode. The reference electrode (12-mm diameter, Tangible Solutions) was placed on the rat's shaved chest with SignaGel as the conducting medium. Once the electrodes were in place, the animal was wrapped with a flexible cohesive bandage (PetFlex, Med-Vet) and placed into their home cage. Anodal tDCS was then applied at $0.25 \mathrm{~mA}$ using a constant-current stimulator (Magstim DCstimulator; Neuroconn) for $30 \mathrm{~min}$. The sham group was prepared the same way as the stimulation groups but did not receive any current (Fig. 1).

\section{Behavioral tests}

To assess memory acquisition and recall following tDCS, a modified passive avoidance test (PAT) was run as a learning and memory test (Fig. 1). Before behavioral testing, animals were allowed to acclimate to the testing room for at least $10 \mathrm{~min}$. On day 1 (habituation), animals had their midsection wrapped in Petflex tape and were returned to their home cage for $20 \mathrm{~min}$. They were then unwrapped and placed into an open arena and allowed to explore freely for $5 \mathrm{~min}$. Immediately following exploration, animals were then taken to the PAT chamber and allowed to freely explore both rooms for $5 \mathrm{~min}$.

Approximately $24 \mathrm{~h}$ later (training, day 2), animals were wrapped for stimulation and placed in their homecage. Only the group designated to receive stimulation before memory acquisition received anodal tDCS, and the other two groups received sham stimulation. After the 30-min stimulation period, animals were unwrapped and placed in the open arena on the opposite wall facing away from the two identical objects present. They were allowed to freely explore the arena for a total duration of $3 \mathrm{~min}$. They were then taken to the PAT chamber (Gemini, San Diego Instruments, Inc.) and placed into a lit room facing away from the gate that led to a dark chamber. After a 30-s acclimation period, the gate rose, and the animal was given the opportunity to cross over to the dark room. Once the animal crossed over, the gate was immediately closed, and after $3 \mathrm{~s}$ the rat received $0.75-\mathrm{mA}$ shock to the feet for $1 \mathrm{~s}$. After the shock was administered, the rat remained in the dark room for $30 \mathrm{~s}$ before being removed and returned to its homecage.

Approximately $24 \mathrm{~h}$ later (testing, day 3 ), the animals were wrapped for stimulation and placed in their homecage. Only rats designated to receive anodal tDCS before memory recall were stimulated, and all of the other rats received sham stimulation. After the stimulation period, animals were unwrapped and placed into the same arena as the previous $2 \mathrm{~d}$ and allowed to explore the arena for $3 \mathrm{~min}$. During this phase, one of the objects was randomly replaced with a different object that the rodent had not been previously exposed to. The different object was placed and rotated between animals. After the 3-min 
exploration, the rodent was then placed into the PAT lit room chamber facing away from the gate and allowed to acclimate for $30 \mathrm{~s}$. The gate separating the light room from the dark room was then lifted and the animal was again given the opportunity to cross into the dark room for a period of $10 \mathrm{~min}$ (600 s). After the animal either crossed over to the dark room or the 10 min maximum time was reached, the animal was removed from the chamber and immediately euthanized by decapitation for brain tissue collection.

\section{Tissue handling}

Tissue preparation followed procedures described previously (Stafford et al., 2018). Briefly, hippocampal tissue from each rat was collected, frozen and stored at $-80^{\circ} \mathrm{C}$. The hippocampal tissues were prepared following the Syn-PER protein extraction method described by the manufacturer (Thermo Fisher Scientific), which yielded two protein fractions: cytosolic and synaptic fraction. The concentration of the synaptic proteins (synaptoneurosomes) was assessed for each sample in duplicates, using the bicinchoninic acid assay (Thermo Fisher Scientific) according to manufacturer's instructions.

\section{Proteomic sample preparation}

Synaptoneurosome isolations $(15 \mu \mathrm{l})$ were suspended in $15 \mu \mathrm{l}$ of $0.2 \%$ Rapigest surfactant (Waters) in $50 \mathrm{mM}$ ammonium bicarbonate supplemented with MS-SAFE protease/phosphatase inhibitor cocktail (Sigma-Aldrich). Protein samples were reduced with dithiothreitol $(5 \mathrm{mM}$ final concentration, Sigma-Aldrich) at $95^{\circ} \mathrm{C}$ for $5 \mathrm{~min}$. Samples were cooled and cysteines were alkylated with iodoacetamide (15 mM final concentration, SigmaAldrich) at ambient temperature for $30 \mathrm{~min}$ in the dark, followed by the protein digestion with $800 \mathrm{ng}$ of sequencing grade modified trysin/Lys-C (Promega Corporation) at $37^{\circ} \mathrm{C}$ overnight with gentle shaking. Neat formic acid (Pierce, Thermo Fisher Scientific) was added to each sample $(50 \% \mathrm{v} / \mathrm{v})$ and samples were returned to $37^{\circ} \mathrm{C}$ for 30 min. Precipitated Rapigest was removed by removing the supernatant following centrifugation at $30,000 \times g$ for 15 min. Centrifugation was repeated until no precipitate remained. Samples were dried in a speed vac and resuspended in $2 \%$ acetonitrile: $0.03 \%$ trifluoroacetic acid (TFA; aq, LC loading buffer). The 280-nm absorbance was used to estimate peptide concentration (Nanodrop) and samples were diluted to $0.5 \mu \mathrm{g} / \mu \mathrm{l}$ in LC loading buffer.

\section{Bottom-up liquid chromatography mass spectrometry (LC-MS/MS)}

All separations were performed on a Dionex Ultimate 3000 RSLCnano liquid chromatography system (Thermo Fisher Scientific). Briefly, digested peptides $(1 \mu \mathrm{g})$ were preconcentrated on a $5 \mu, 100 \AA, 300 \mu \mathrm{m} \times 5 \mathrm{~mm} \mathrm{C18}$ PepMap 100 trap column (Thermo Fisher Scientific) using LC loading buffer under isocratic conditions at $5 \mu \mathrm{l} \mathrm{\textrm {min } ^ { - 1 }}$ for $7.5 \mathrm{~min}$. Peptides were reversed-phase separated on an Easy-Spray PepMap $3 \mu \mathrm{m}, 100 \AA \AA, 75 \mu \mathrm{m} \times 15 \mathrm{~cm}$

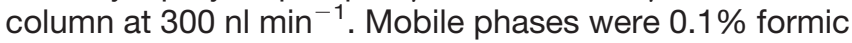
acid (aq, A) and $0.1 \%$ formic acid in acetonitrile (B, Optima MS Grade, Thermo Fisher Scientific). Analytical sep- arations were conducted over $180 \mathrm{~min}$ at $3 \% \mathrm{~B}$ for $10 \mathrm{~min}$, $30 \%$ B for $152 \mathrm{~min}, 40 \%$ B at $157 \mathrm{~min}$ followed by a 10 min wash at $90 \% \mathrm{~B}$, and a $10-$ min equilibration at $3 \% \mathrm{~B}$. Eluted peptides were introduced into an Orbitrap Fusion Lumos mass spectrometer equipped with Easy-Spray source operated at $2.2 \mathrm{kV}$ (Thermo Fisher Scientific). MS ${ }^{1}$ scans were acquired at 120,000 resolution across $375-$ $2000 \mathrm{~m} / \mathrm{z}$ using the Easy-IC reagent (fluoranthene) for internal mass calibration. Precursors were selected based on a $\mathrm{MS}^{(n-1)}$ scans and isolated for data-dependent $\mathrm{MS}^{n}$ scans in the quadrapole operated with $1.2 \mathrm{~m} / \mathrm{z}$ isolation window. Fragments were generated by collision-induced dissociation (CID) with a 10-ms activation time and a $35 \%$ normalized collision energy for +2 to +7 precursor charges states in the ion trap using all available parallelizable time over 2-s cycles. Dynamic exclusion for $\mathrm{MS}^{n}$ scans was set at a \pm 10 ppm mass tolerance with exclusion occurring after one time for $15 \mathrm{~s}$.

\section{Proteomic data processing}

Proteomic data were searched using the Proteome Discoverer software suite (v. 2.2) equipped with the Sequest HT search engine (Thermo Fisher Scientific). Briefly, tandem data were searched against the Uniprot reviewed Rattus norvegicus (as of 250ct17) and the common Repository of Adventitious Proteins (cRAP) databases. Search parameters were as follows: MS tolerance 10 $\mathrm{ppm}$, an $\mathrm{MS}^{n}$ tolerance of $0.5 \mathrm{Da}$, and three allowed missed tryptic cleavages. Amino acid modifications searched were carbamidomethyl of cysteine (static), oxidation of methionine (dynamic), and acetylation of the $\mathrm{N}$ terminus (dynamic). Peptide spectral matches (PSMs) were evaluated using the target decoy PSM validator with a maximum $\mathrm{Cn}$ of 0.05 and a decoy database search with a target false discovery rate (FDR, $q$ value) of 0.05 (relaxed) and 0.01 (strict). FDR was estimated with 0.05 (relaxed) and 0.01 (strict) thresholds. Precursor ion label free quantitation was performed on unique and razor peptides with retention time alignment of $<10 \mathrm{~min}$, mass tolerance $10 \mathrm{ppm}$, abundance based on intensity, and data normalized to total peptide amount. Normalized abundances were exported from Proteome Discoverer for further downstream analysis described below.

\section{Data analysis and statistical methods}

Behavioral data from the PAT consisted of latency variables that were analyzed by two-way repeated measures ANOVA with the Holm-Sidak post hoc method and Cox proportional hazard regression test. A homoscedastic two-tailed Student's $t$ test was conducted to identify statistically differentially expressed proteins (DEPs) of normalized abundance variables between treatment groups. Principal component analysis (PCA) on the correlation matrix with the default estimation method was employed to statistically explain the structure of normalized abundance data across groups. Hierarchical clustering analysis with the distance method of Ward to calculate distances between all pairs of clusters was performed. For these data analyses, the statistical software SigmaPlot (version 12.3, Systat Software, Inc.), JMP Pro (version 
13.2, SAS Institute Inc.), and Microsoft Excel 2013 were used.

The enrichment and pathway analysis on DEPs of the normalized abundance data sets were performed with the Panther (Protein ANalysis THrough Evolutionary Relationships) classification system (Mi et al., 2013) and the Database for Annotation, Visualization and Integrated Discovery (DAVID) Bioinformatics resources (Huang da et al., 2009). The DAVID Bioinformatics resources was also used to perform a functional annotation clustering analysis for DEPs of the normalized abundance data sets. The DEPs were also analyzed by the Ingenuity Pathway Analysis (IPA; QIAGEN) to identify the diseases and biological functions that are associated with behaviors. The Ingenuity Upstream Regulator analysis in IPA was performed to identify the upstream transcriptional regulators in addition to conducting the IPA's network analysis.

Protein-protein interaction (PPI) network analysis for the organism Rattus norvegicus (network edge setting: molecular action) was conducted for DEPs by using the STRING database (Szklarczyk et al., 2017). The Markov cluster (MCL) algorithm with an inflation parameter of 3 was used if needed. Protein sequence similarity (PSS) network analysis for the organisms Rattus norvegicus was also performed. The BLASTP suite (BLASTP 2.8.0+) was used to search proteins and determine their sequence similarity. Cytoscape (version 3.7) was used to build PSS network visualization and conduct network analysis (Shannon et al., 2003). The Cytoscape plugin app ClusterONE was used to cluster with overlapping neighborhood expansion to detect potentially overlapping protein complexes from the interaction data (Nepusz et al., 2012). Centrality measures were calculated to identify critical proteins that play a central role in mediating interactions among given proteins in PPI and PSS networks (Jeong et al., 2001; Ashtiani et al., 2018) by using CytoNCA (Wang et al., 2012). The GeneMANIA was also used to identify the function of molecules in networks (Montojo et al., 2010; Warde-Farley et al., 2010) as needed.

\section{Data availability}

Proteomics data are provided as Extended Data Figure 1-3.

\section{Results}

\section{PAT}

The PAT was used to examine the effect of anodal tDCS administered before memory acquisition or memory recall on cognitive performance. Two-way repeated measures ANOVA (session $\times$ group) was performed for PAT latency variable. A significant trial difference $(F=25.05, p<$ 0.001 ) was detected (Fig. $2 A$ ). All groups resulted in statistical significance between training and testing days, confirming that the learning experience of PAT was significantly completed by all animals across treatment groups. Additionally, on the testing day, the post hoc test shows that the acquisition group significantly performed better on PAT when compared to the sham group $(p=$ 0.046), but no significant difference between sham and retrieval groups was detected. However, because the two-way repeated measures ANOVA did not detect a statistical significance from the treatment group variation $(F=1.695, p=0.196)$, Cox proportional hazard regression test was also conducted for PAT data on training and testing days (Fig. 2B,C). The result first confirmed no group difference during the training day. However, on the testing day, there is a significant difference between the acquisition and sham groups indicating that brain stimulation before training enhances memory performance $\left(\chi^{2}\right.$ $p=0.0445$, risk ratio $=2.306331$ )

\section{Overall results of hippocampal synaptic proteomics}

Proteomics analysis was conducted to determine the underlying effects of brain stimulation on protein abundance in the rat hippocampus, an area of the brain associated with learning and memory. The proteomics data were first validated by comparing the protein abundances within each group (replicability; Extended Data Fig. 1-1) and analyzing the abundance levels of 16 proteins that are usually used as internal control (Extended Data Fig. 1-2). All 16 proteins show no group difference between sham and acquisition groups, and 15 proteins resulted in no difference between sham and retrieval groups. The abundance levels of 3687 proteins were detected, but 2909 proteins were used for further analyses because 778 proteins contain a value of zero for the most samples $(\geq 35$ samples out of 42 samples; Extended Data Fig. 1-3). When compared to the sham group, the acquisition and retrieval groups resulted in 184 and 82 significant proteins, respectively ( $p<0.0500$, two-tailed test). Interestingly, 431 proteins were significantly identified when the two tDCS groups were compared. PCA showed a clear group separation between sham and acquisition groups, but the retrieval group overlapped with the other groups (Fig. 2D). The hierarchical clustering analysis resulted in the similar pattern to the PCA result (Fig. 2E).

\section{Gene ontology (GO) enrichment analysis and pathway analysis (PantherDB and DAVID Bioinformatics)}

GO enrichment analysis and pathway analysis were performed to investigate the effects of tDCS on synaptoneurosomes and pathways in the rat hippocampus. Using lists of significantly, DEPs between two groups, GO enrichment analysis was performed by using PantherDB and DAVID Bioinformatics. The analysis from the comparison between sham and retrieval groups resulted in only three cellular component (CC) and two pathways. The two pathways are TGF- $\beta$ signaling pathway (P00052, FDR $q=$ $0.026)$ and gonadotropin-releasing hormone receptor pathway (P06664, FDR $q=0.00223$ ). The analysis of Panther GO slim with significant genes between sham and acquisition groups (Table 1) resulted in 12 CC, 20 biological processes (BPs), 16 molecular functions (MFs), and seven pathways. The GO slim results showed that the significant genes are significantly associated with neuronal synaptic membrane and protein complex (from CC results); neuron-neuron synaptic transmission and transport (from BP results); and significantly connected to the signaling pathways of glutamate receptor and voltagegated ion channel activities (from MF results). The results 


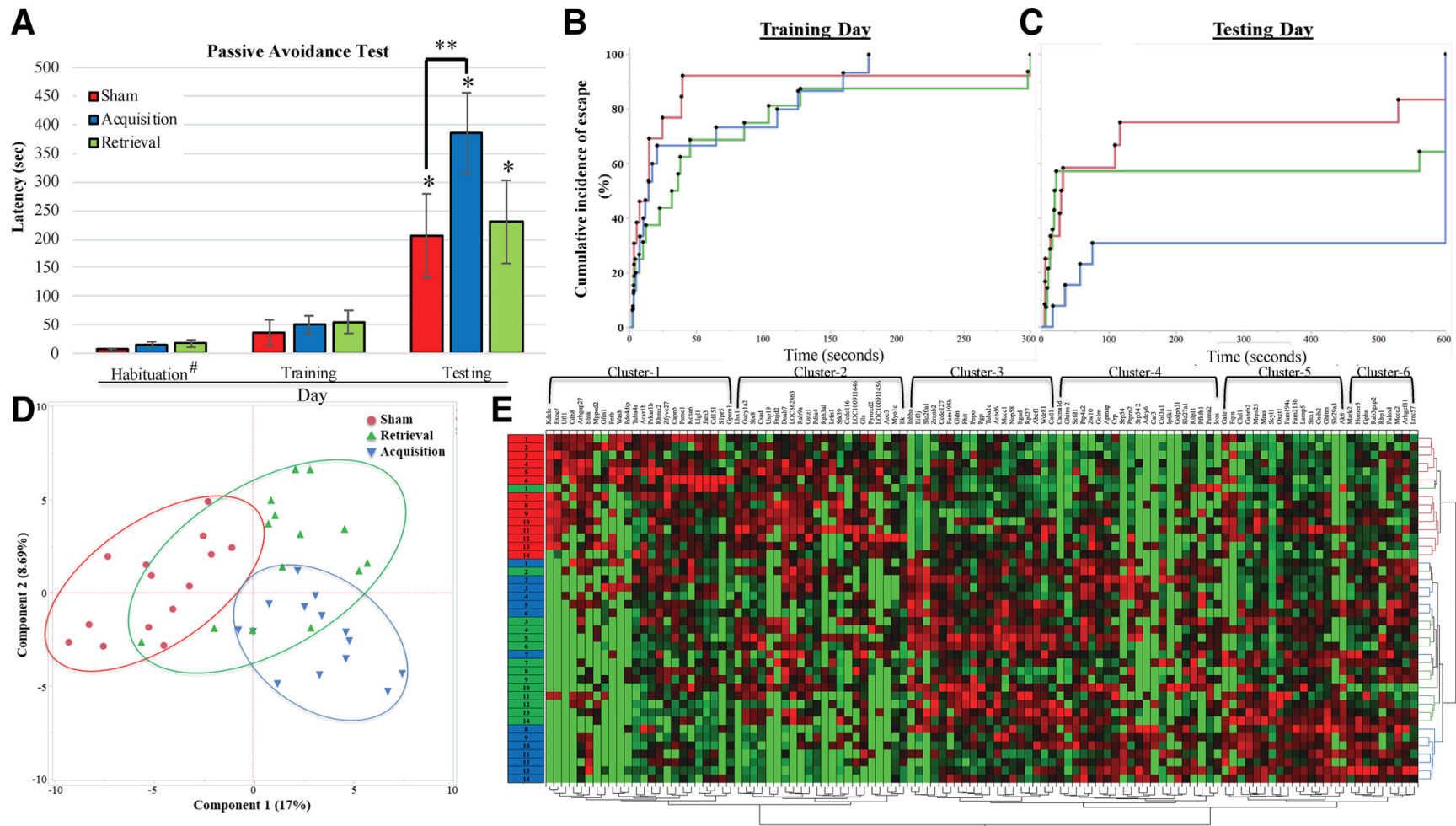

Figure 2. Behavioral data analysis. A, Analysis of passive avoidance data. Two-way RM ANOVA detected a significant difference in the latency of all groups between the training and testing days. During the testing day, a statistical significance was detected between sham and acquisition groups. \#Habituation data were collected from only eight animals per group; * and ** indicate statistical significance $(p<0.05$ and $p<0.001$, respectively). $\boldsymbol{B}$, Cox proportional hazard regression analysis for PAT data on training day. No statistical group separation was detected. $\boldsymbol{C}$, Cox proportional hazard regression analysis for PAT data on testing day. A significant group difference was detected (log-rank $\mathrm{X}^{2}=7.0919, \mathrm{df}=2, p=0.0288$ ). $\boldsymbol{D}, \mathrm{PCA}$ with proteomics data show the distribution of each samples across the groups. $\boldsymbol{E}$, Hierarchical clustering analysis with proteomics data show the distribution of each sample across the groups. Red, green, and blue boxes represent sham, retrieval, and acquisition samples. Protein IDs in the first hierarchical clustering group, from left to right, are KDELC2, EXOC6, UFL1, CDH8, ARHGAP27, BLNK, MPPED2, GFM1, FNTB, WASH, PDE4DIP, TUBA4A, ACVR1B, PRKAR1B, RBMX2, ZFYVE27, CAPN5, PSME1, KCNA6, LLGL1, JAM3, CD151, S1PR5, and GPSM1. The hierarchical clustering group 2 includes, from left to right, LHX1, GUCY1A2, STX8, CSAD, USP19, FTSJD2, DNAH7, LOC362863, RAB9A, GSTZ1, PDIA4, RPH3AL, LRFN1, STK39, CCDC116, LOC100911646, GLS, PYROXD2, LOC100911456, AOC3, MYO1C, and ILK. For the cluster group 3, protein IDs are INHBA, EIF3J, SLC20A1, ZRANB2, CCDC127, FAM195B, GLDN, FHIT, PNPO, PGP, TUBA1C, ACBD6, MCCC1, NOP58, ITGAD, RPL27, ABCF1, WDR81, and COTL1. The cluster group 4 contains proteins named as, from left to right, CACNA1D, GHITM2, SCFD1, PTP4A2, ZW10, GCLM, APMAP, CRP, SRP54, PTPRN2, SRP542, ADCY6, CAR3, COL3A1, IP6K1, GOLPH31, SLC27A1, RILPL1, PFKFB1, PSMA2, and ICOS. Proteins from the cluster group 5 are GALE, EQTN, CLUL1, GABRB2, MRPS25, MRAS, SCYL1, OXCT1, FAM194A, FAM213B, LAMP5, SNX1, CNIH2, GHITM, SLC29A3, and AK6. Proteins in the cluster group 6 are MARK2, HOMER3, GPHN, RAB3GAP2, RBP1, PALMD, MCCC2, ARHGEF11, and LRRC57. For a clear figure, see Extended Data Figure 2-1.

of the Panther pathways analysis include glutamate receptor pathways (P00037, P00041, and P00039) and beta-adrenergic receptor pathways (P04378 and P04377). Moreover, the Reactome pathway analysis shows that the significant genes identified from the comparison between sham and acquisition groups are significantly involved in 20 different pathways (Extended Data Table 1-1). Among them, the most abundant pathways are receptor signaling, including NMDA receptor, glutamate receptor, and ion channels. From the significant genes between sham and retrieval groups, however, no significant pathways and GO slim terms for BP and MF were detected. Only three GO slim CCs were detected and they are: cytoplasm (GO:0005737, fold change (FC): 2.39), intracellular (GO: 0005622, FC: 1.71), and cell part (GO:0044464, FC: 1.68) with $q$ values from FDR analysis $<0.05$. The significant gene list identified from the comparison between the acquisition and recall groups resulted in $20 \mathrm{CC}, 42 \mathrm{BP}$, and 26 MF with a statistical significance from the FDR analysis (Table 2; Extended Data Table 2-1). The CC terms identified by the GO slim analysis includes postsynaptic membrane, synapse, SNARE complex, neuron projection and protein complex $(q<0.05)$. Growth, neuronneuron synaptic transmission, and different transports and metabolisms were detected for the BP terms $(q<$ 0.05); while glutamate receptor activity, SNAP receptor activity and multiple kinase activities were identified for the MF terms $(q<0.05)$.

The functional annotation clustering analysis provided by the DAVID bioinformatic database resulted in 54 annotation clusters for the comparison between sham and acquisition groups. Among them, 11 annotation clusters 
Table 1. Results of PantherDB analysis for the comparison between sham and acquisition groups

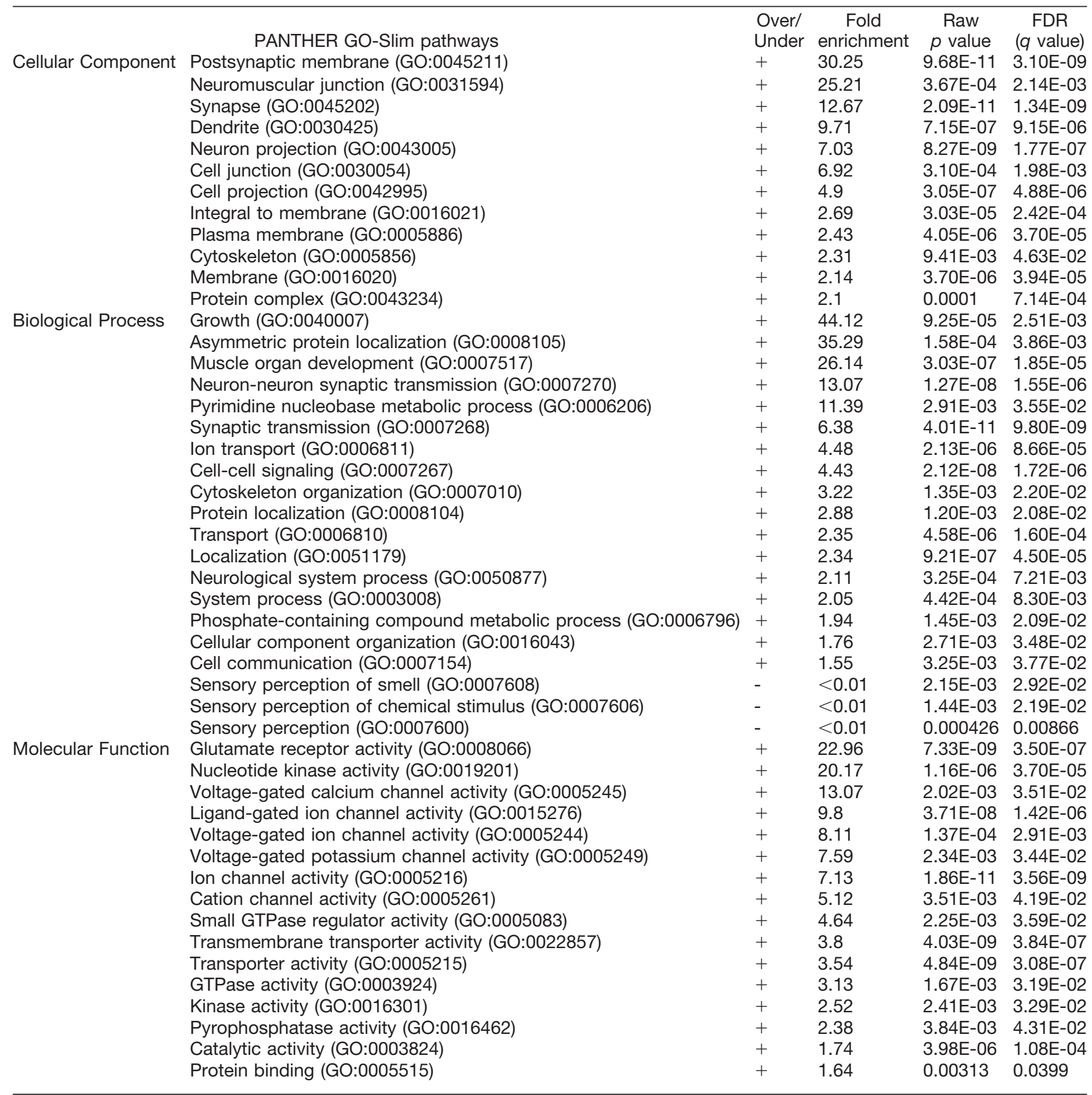

* See Extended Data Table 1-1 for all Reactome pathway terms identified from the comparison between sham and acquisition groups.

have an enrichment score $>4.0$ (Table 3; Extended Data Table 3-1). The functional clustering annotation terms "postsynaptic membrane" and "synapse" resulted in the highest enrichment scores (13.96 and 12.4, respectively). Moreover, the results also contain the terms regulation of excitatory postsynaptic potential, glutamate receptor, NMDA receptor, and neuronal membrane-associated guanylate-kinase-associated proteins. However, the functional annotation clustering analysis for the comparison between sham and retrieval groups did not result in any significant FDR (the lowest $q=1.72$ ) with the highest enrichment score of 1.97; thus, no further analysis was considered.

\section{IPA}

IPA was conducted to identify the diseases and biological functions that are associated with behavior. From the comparison between retrieval and sham groups, functions of locomotion $(p=0.00028)$ and working $(p=$ 0.00265) were detected (Extended Data Fig. 3-1). More behavior-associated functional annotations were detected from the comparison between the acquisition and 
Table 2. Results of PantherDB analysis for the comparison between sham and retrieval groups

\begin{tabular}{|c|c|c|c|c|c|}
\hline Cellular & $\begin{array}{r}\text { PANTHER GO-Slim pathways } \\
\text { Postsynaptic membrane (GO:0045211) }\end{array}$ & $\begin{array}{l}\text { Over/ } \\
\text { Under } \\
+\end{array}$ & $\begin{array}{l}\quad \text { Fold } \\
\text { enrichment } \\
14.71\end{array}$ & $\begin{array}{c}\text { Raw } \\
p \text { value } \\
9.98 \mathrm{E}-09\end{array}$ & $\begin{array}{c}\text { FDR } \\
\text { (q value) } \\
7.99 \mathrm{E}-08\end{array}$ \\
\hline \multirow[t]{19}{*}{ Component } & Neuromuscular junction (GO:0031594) & + & 11.03 & 3.83E-03 & 1.29E-02 \\
\hline & Proton-transporting ATP synthase complex (GO:0045259) & + & 9.8 & $1.20 \mathrm{E}-03$ & 4.51E-03 \\
\hline & Synapse (GO:0045202) & + & 8.31 & $1.24 \mathrm{E}-12$ & $7.96 \mathrm{E}-11$ \\
\hline & Mitochondrial inner membrane (GO:0005743) & + & 7.1 & 4.77E-09 & $5.08 \mathrm{E}-08$ \\
\hline & Dendrite (GO:0030425) & + & 7.08 & $1.49 \mathrm{E}-08$ & 1.06E-07 \\
\hline & SNARE complex (GO:0031201) & + & 6.06 & $5.75 E-03$ & 1.84E-02 \\
\hline & Neuron projection (GO:0043005) & + & 5.33 & $2.61 \mathrm{E}-11$ & $5.57 \mathrm{E}-10$ \\
\hline & Cell junction (GO:0030054) & + & 5.05 & 5.70E-05 & 2.60E-04 \\
\hline & Axon (GO:0030424) & + & 4.68 & $1.31 \mathrm{E}-02$ & $3.98 \mathrm{E}-02$ \\
\hline & Cell projection (GO:0042995) & + & 3.75 & $7.41 \mathrm{E}-09$ & 6.77E-08 \\
\hline & Protein complex (GO:0043234) & + & 2.31 & 8.91E-12 & $2.85 \mathrm{E}-10$ \\
\hline & Cytoskeleton (GO:0005856) & + & 2.2 & $5.35 \mathrm{E}-04$ & $2.14 \mathrm{E}-03$ \\
\hline & Membrane (GO:0016020) & + & 1.99 & 1.14E-09 & 1.46E-08 \\
\hline & Integral to membrane (GO:0016021) & + & 1.87 & $5.15 E-04$ & 2.20E-03 \\
\hline & Macromolecular complex (GO:0032991) & + & 1.79 & $2.02 \mathrm{E}-07$ & 1.17E-06 \\
\hline & Cytoplasm (GO:0005737) & + & 1.76 & $5.38 \mathrm{E}-10$ & 8.61E-09 \\
\hline & Plasma membrane (GO:0005886) & + & 1.59 & $1.62 \mathrm{E}-03$ & 5.77E-03 \\
\hline & Cell part (GO:0044464) & + & 1.44 & $1.96 \mathrm{E}-07$ & 1.25E-06 \\
\hline & Intracellular (GO:0005622) & + & 1.37 & $2.18 \mathrm{E}-05$ & 1.07E-04 \\
\hline Biological & Growth (GO:0040007) & + & 19.3 & $1.01 \mathrm{E}-03$ & $8.82 \mathrm{E}-03$ \\
\hline \multirow[t]{19}{*}{ Process* } & Asymmetric protein localization (GO:0008105) & + & 15.44 & $1.70 \mathrm{E}-03$ & $1.34 \mathrm{E}-02$ \\
\hline & Oxidative phosphorylation (GO:0006119) & + & 10.89 & 2.67E-08 & $9.31 \mathrm{E}-07$ \\
\hline & Pyrimidine nucleobase metabolic process (GO:0006206) & + & 8.3 & $5.69 \mathrm{E}-04$ & $6.31 \mathrm{E}-03$ \\
\hline & JNK cascade (GO:0007254) & + & 8.04 & $6.47 \mathrm{E}-04$ & $6.32 \mathrm{E}-03$ \\
\hline & Respiratory electron transport chain (GO:0022904) & + & 7.35 & 3.03E-09 & 1.48E-07 \\
\hline & Generation of precursor metabolites and energy (GO:0006091) & + & 6.03 & $1.52 \mathrm{E}-11$ & $3.72 \mathrm{E}-09$ \\
\hline & Neuron-neuron synaptic transmission (GO:0007270) & + & 5.72 & 2.13E-05 & 3.25E-04 \\
\hline & Purine nucleobase metabolic process (GO:0006144) & + & 5.63 & 4.04E-04 & 4.93E-03 \\
\hline & Glycolysis (GO:0006096) & + & 5.51 & $1.15 \mathrm{E}-03$ & $9.39 \mathrm{E}-03$ \\
\hline & Mitochondrial transport (GO:0006839) & + & 5.48 & 3.06E-03 & 1.86E-02 \\
\hline & Neurotransmitter secretion (GO:0007269) & + & 4.98 & $1.46 \mathrm{E}-04$ & $1.98 \mathrm{E}-03$ \\
\hline & Cation transport (GO:0006812) & + & 4.24 & $1.89 \mathrm{E}-03$ & $1.44 \mathrm{E}-02$ \\
\hline & Calcium-mediated signaling (GO:0019722) & + & 4.14 & $2.14 \mathrm{E}-03$ & $1.45 \mathrm{E}-02$ \\
\hline & Mitochondrion organization (GO:0007005) & + & 4 & $2.56 \mathrm{E}-03$ & 1.65E-02 \\
\hline & Synaptic transmission (GO:0007268) & + & 3.99 & $5.54 \mathrm{E}-10$ & $4.51 \mathrm{E}-08$ \\
\hline & Anatomical structure morphogenesis (GO:0009653) & + & 3.68 & $6.22 \mathrm{E}-04$ & 6.60E-03 \\
\hline & Protein targeting (GO:0006605) & + & 3.47 & $5.29 E-04$ & $6.15 \mathrm{E}-03$ \\
\hline & Ion transport (GO:0006811) & + & 2.87 & $1.82 \mathrm{E}-05$ & 2.96E-04 \\
\hline & Cell-cell signaling (GO:0007267) & + & 2.86 & $3.92 \mathrm{E}-07$ & $9.58 \mathrm{E}-06$ \\
\hline Molecular & Glutamate receptor activity (GO:0008066) & + & 11.3 & $3.82 \mathrm{E}-07$ & $9.12 \mathrm{E}-06$ \\
\hline \multirow[t]{14}{*}{ Function* } & Nucleotide kinase activity (GO:0019201) & + & 10.29 & $1.32 \mathrm{E}-05$ & 1.94E-04 \\
\hline & SNAP receptor activity (GO:0005484) & + & 7.35 & 3.07E-03 & 2.66E-02 \\
\hline & Hydrogen ion transmembrane transporter activity (GO:0015078) & + & 7.05 & $4.00 \mathrm{E}-06$ & $8.48 \mathrm{E}-05$ \\
\hline & Carbohydrate kinase activity (GO:0019200) & + & 6.64 & 4.27E-03 & 3.26E-02 \\
\hline & Anion channel activity (GO:0005253) & + & 5.25 & $3.61 \mathrm{E}-03$ & 2.87E-02 \\
\hline & Ligand-gated ion channel activity (GO:0015276) & + & 5.07 & 4.31E-06 & 8.24E-05 \\
\hline & Voltage-gated ion channel activity (GO:0005244) & + & 4.14 & $2.14 \mathrm{E}-03$ & 2.27E-02 \\
\hline & Ion channel activity (GO:0005216) & + & 4.06 & 5.90E-09 & 3.76E-07 \\
\hline & Cation channel activity (GO:0005261) & + & 3.58 & 2.49E-03 & 2.51E-02 \\
\hline & Microtubule binding (GO:0008017) & + & 3.4 & $5.95 \mathrm{E}-03$ & 4.37E-02 \\
\hline & Small GTPase regulator activity (GO:0005083) & + & 3.39 & $1.12 \mathrm{E}-03$ & 1.34E-02 \\
\hline & Oxidoreductase activity (GO:0016491) & + & 3 & $5.89 \mathrm{E}-08$ & $1.88 \mathrm{E}-06$ \\
\hline & Kinase activity (GO:0016301) & + & 2.79 & $2.71 \mathrm{E}-07$ & 7.39E-06 \\
\hline & Calcium ion binding (GO:0005509) & + & 2.79 & $2.80 \mathrm{E}-03$ & 2.54E-02 \\
\hline
\end{tabular}

*See Extended Data Table 2-1 for all GO-Slim pathway terms.

sham groups (Fig. 3A; Extended Data Fig. 3-2). Interestingly, functions of memory, cognition and learning were significantly enhanced in the acquisition group while grooming, anxiety and emotional behavior were slightly, but significantly reduced. Statistically significant diseases and functions detected from the comparison between retrieval and sham groups include angiogenesis, apoptosis, necrosis, cognitive impairment and development of 
Table 3. Results of DAVID Bioinformatics analysis for the comparison between sham and acquisition groups

\begin{tabular}{|c|c|c|c|c|c|c|}
\hline \multirow{3}{*}{$\begin{array}{l}\text { Cluster } \\
1\end{array}$} & \multirow[b]{2}{*}{ Functional annotation term summary* } & \multirow{2}{*}{$\begin{array}{l}\text { Enrichment } \\
\text { score }\end{array}$} & \multicolumn{4}{|c|}{ FDR ( $q$ value) } \\
\hline & & & Median & SD & Lowest & Highest \\
\hline & Postsynaptic membrane & 13.96 & $5.88 \mathrm{E}-13$ & 0.055 & $1.65 E-20$ & 0.18 \\
\hline 2 & Synapse & 12.40 & $6.63 \mathrm{E}-12$ & 0.041 & $1.65 E-20$ & 0.092 \\
\hline 3 & Ion transport & 9.54 & $3.25 E-07$ & 4.75E-07 & 1.49E-07 & $1.05 \mathrm{E}-06$ \\
\hline 4 & Mitochondrial inner membrane & 7.44 & 0.0154 & 0.093 & $2.25 E-12$ & 0.19 \\
\hline 5 & Positive regulation of excitatory postsynaptic potential & 5.23 & 0.0014 & 0.848 & 3.79E-04 & 1.47 \\
\hline 6 & Neuronal membrane-associated guanylate kinases & 5.16 & 0.0163 & 0.224 & 4.25E-04 & 0.46 \\
\hline 7 & PDZ and $\mathrm{SH} 3$ domains & 4.91 & 0.0121 & 19.78 & $1.44 \mathrm{E}-07$ & 65.75 \\
\hline 8 & Ionotropic glutamate and NMDA receptors & 4.86 & 0.0272 & 0.859 & 3.01E-04 & 1.50 \\
\hline 9 & AMPA glutamate receptor complex & 4.42 & 0.0104 & 5.28 & 0.0018 & 9.15 \\
\hline 10 & Glutamatergic synapses & 4.34 & 0.1636 & 25.26 & $1.44 \mathrm{E}-07$ & 100.0 \\
\hline 11 & Guanylate-kinase-associated protein & 4.25 & 1.0843 & 0.682 & 4.25E-04 & 1.26 \\
\hline
\end{tabular}

* See Extended Data Table 3-1 for detailed functional clustering annotation terms.

neurons (activation $z$-scores: $-2.404,-2.205,-2.159$, -1.941 , and 1.514 , respectively). tDCS before memory acquisition significantly enhanced plasticity of synapse, neurotransmission, synaptic transmission, synaptic transmission of nervous tissue, synaptic transmission of hippocampal cells, quantity of synaptic vesicles, function of neurons, branching of neurites, neuritogenesis, and longterm potentiation (LTP; activation z-scores: 2.36, 2.718, $2.701,2.67,2.762,2.0,2.0,1.538,1.291$, and 1.259, respectively). Additionally, the acquisition group resulted in significant decreases in neurologic diseases, including hyperactive disorder, seizures, movement disorder, neurodegeneration, and cognitive impairment (activation zscores: $-3.679,-2.788,-2.015,-1.125$, and -1.0 , respectively).

IPA generated multiple networks (Extended Data Fig. 3-3). Among them, only one network (Fig. 3B) identified from the analysis between retrieval and sham groups was associated with neurologic and psychological disorders. The analysis between the acquisition and sham groups resulted in 6 networks that are associated with the nervous system. When the six networks were merged into a

A

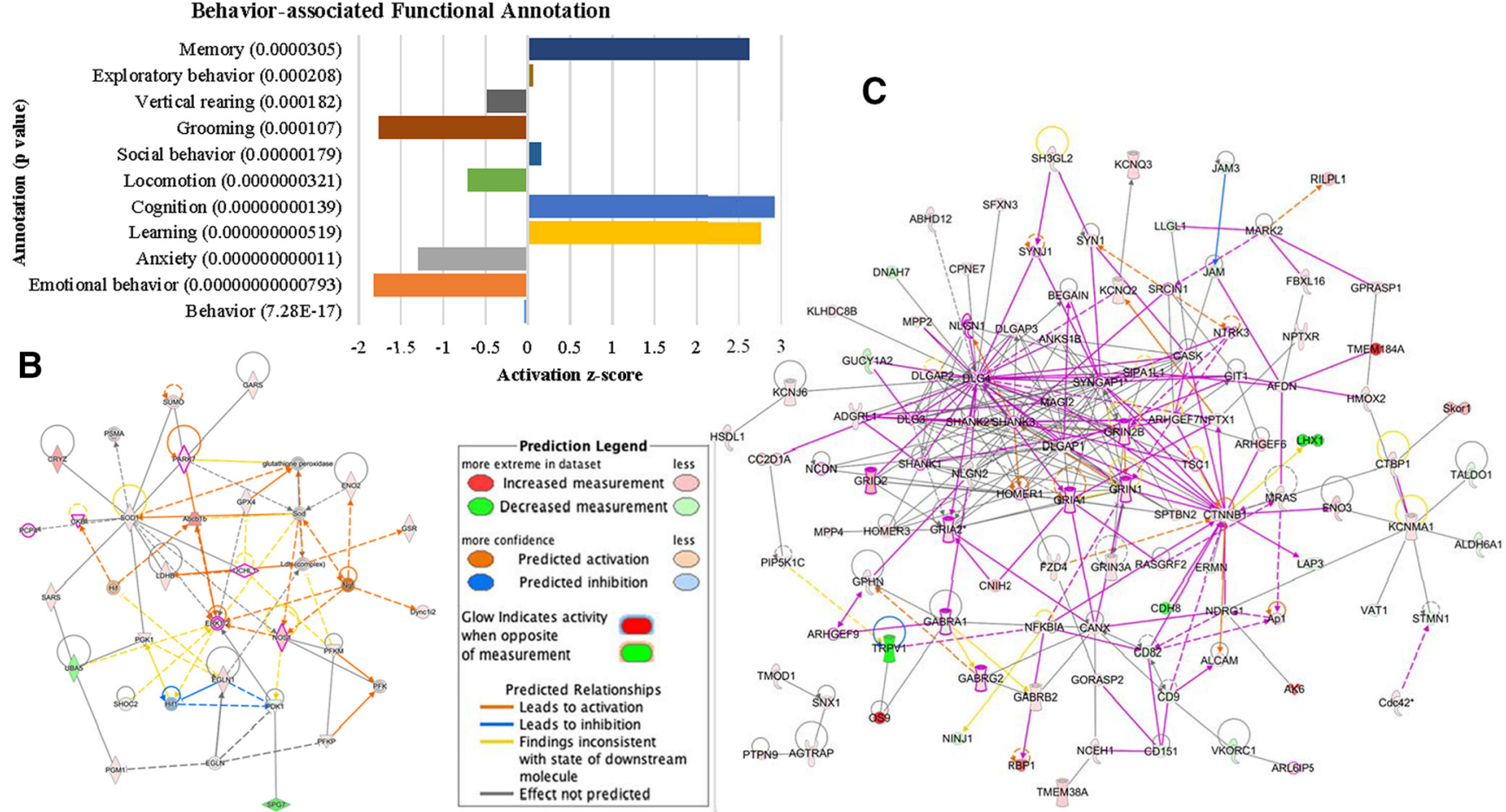

Figure 3. IPA results. A, Behavior-associated functional annotation detected from the comparison between the acquisition and sham groups. $\boldsymbol{B}$, Network-1 detected from the comparison between the retrieval and sham groups. $\boldsymbol{C}$, Six networks that were associated with the nervous system were merged in to a network. Nodes that did not pass cutoff in dataset, not in overlaid dataset, and not connected to any other nodes were excluded from the network. Pink-outlined molecules are associated with cognition ( $p=1.81 \mathrm{E}-3)$. Molecule activity predictor (MAP) was also overlaid to predict the upstream and downstream effects of activation or inhibition on other molecules. For all IPA data, see Extended Data Figures 3-1, 3-2, 3-3, 3-4. 


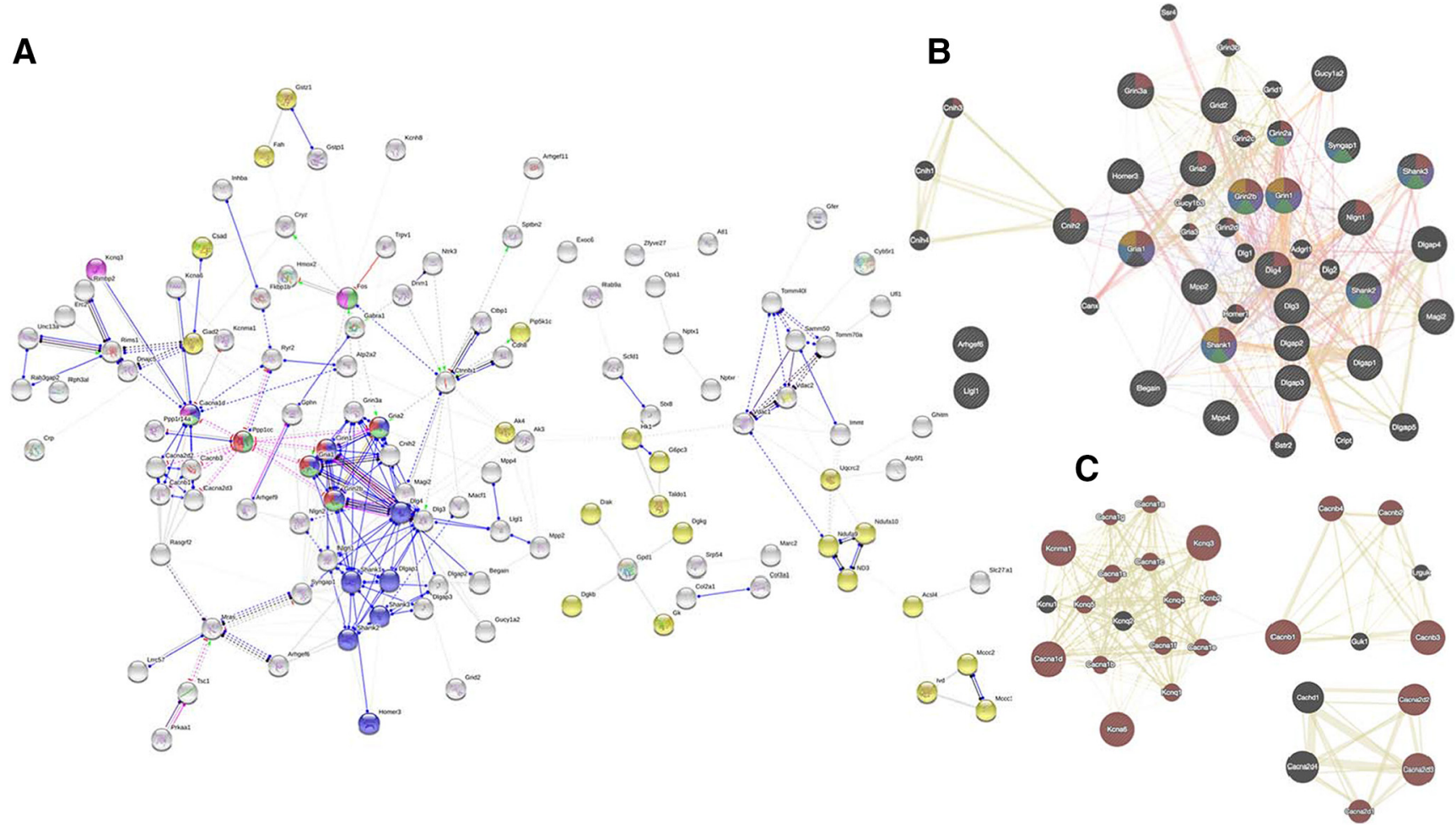

Figure 4. PPI network analysis. $\boldsymbol{A}$, PPI network for the significant molecules from the acquisition-and-sham comparison. Nodes were colored for functions of glutamatergic synapse (blue), metabolic pathways (yellow), dopaminergic synapse (green), LTP (red), and cholinergic synapse (pink). Disconnected nodes were excluded. $\boldsymbol{B}$, The first cluster network of MCL algorithm. $\boldsymbol{C}$, The second cluster network of MCL algorithm.

larger network (Fig. 3C), molecules associated with memory ( $p=1.65 \mathrm{E}-7)$, learning $(p=9.69 \mathrm{E}-16)$, and spatial learning ( $\rho=9.09 \mathrm{E}-7)$ were most prevalent. The effects of top regulators were analyzed (Extended Data Fig. 3-4), but nervous system-associated regulators were not detected from the analysis between retrieval and sham groups. The analysis between the acquisition and sham group identified multiple top regulators that are associated with the nervous system. The network of regulators (APOE, BDNF, GSK3B, and SHANK3) targets 17 molecules in the dataset and are associated with function of neurons, memory, plasticity of synapse and synaptic transmission (consistency score: 8.731). Additionally, two networks including the nervous system regulator BDNF were identified and associated with synaptic transmission (total target molecule \#: 5, consistency scores: -4.919 both). Top upstream regulators were also detected from the ingenuity upstream regulator analysis in IPA. The analysis between retrieval and sham groups identified MAPT, PSEN1, and APP (p values of overlap: 6.80E-16, 7.61E10 , and 3.77E-09, respectively) as top upstream regulators. PSEN1, MAPT1, APP, and L-dopa ( $p$ values of overlap: 2.95E-13, 3.03E-12, 1.04E-08, and 1.47E-08, respectively) were detected as the top upstream regulators for the comparison between the acquisition and sham groups.

\section{Network analysis}

PPI networks were developed and analyzed to identify the effects of stimulation on protein signaling cascades.
The PPI network for the statistically significant molecules detected from the retrieval compared to the sham group was not significant (average node degree: 0.53; average local clustering coefficient: 0.317 ; PPI enrichment $p=$ 0.648). However, the PPI network from the acquisition group compared to the sham group had significant interactions (average node degree: 3.06; average local clustering coefficient: 0.404; PPI enrichment $p<1.0 \mathrm{E}-16$; Fig. $4 A)$. The PPI network was further clustered by the MCL method. The MCL cluster-1 network (Fig. 4B) was significantly related to the glutamate receptor signaling pathway (brown colored nodes; $q=2.68 \mathrm{E}-29$ ), memory (purple colored nodes; $q=5.94 \mathrm{E}-07$ ), learning (green colored nodes; $q=1.097 \mathrm{E}-06$ ), cognition (blue colored nodes; $q=4.98 \mathrm{E}-06$ ), and long-term memory (yellow colored nodes; $q=0.0001$ ). The MCL cluster-2 network (Fig. 4C) was significantly associated with the function of voltage-gated channel activity (brown colored nodes; $q=$ 4.03E-40). Network centralities were calculated for the MCL cluster-1 network to identify candidate molecules that may lethally affect to the network. Based on the subgraph centrality measure, the top five ranked proteins are DLG4, GRIN2a, SHANK1, GRIA1, and DLG3, and the expression of these proteins resulted in significant group difference between the acquisition and sham groups $(p<$ 0.01).

The BLASTP suite (BLASTP 2.8.0+) was used to search for proteins and determine their sequence similarity. PSS network was created by using Cytoscape, and proteins 


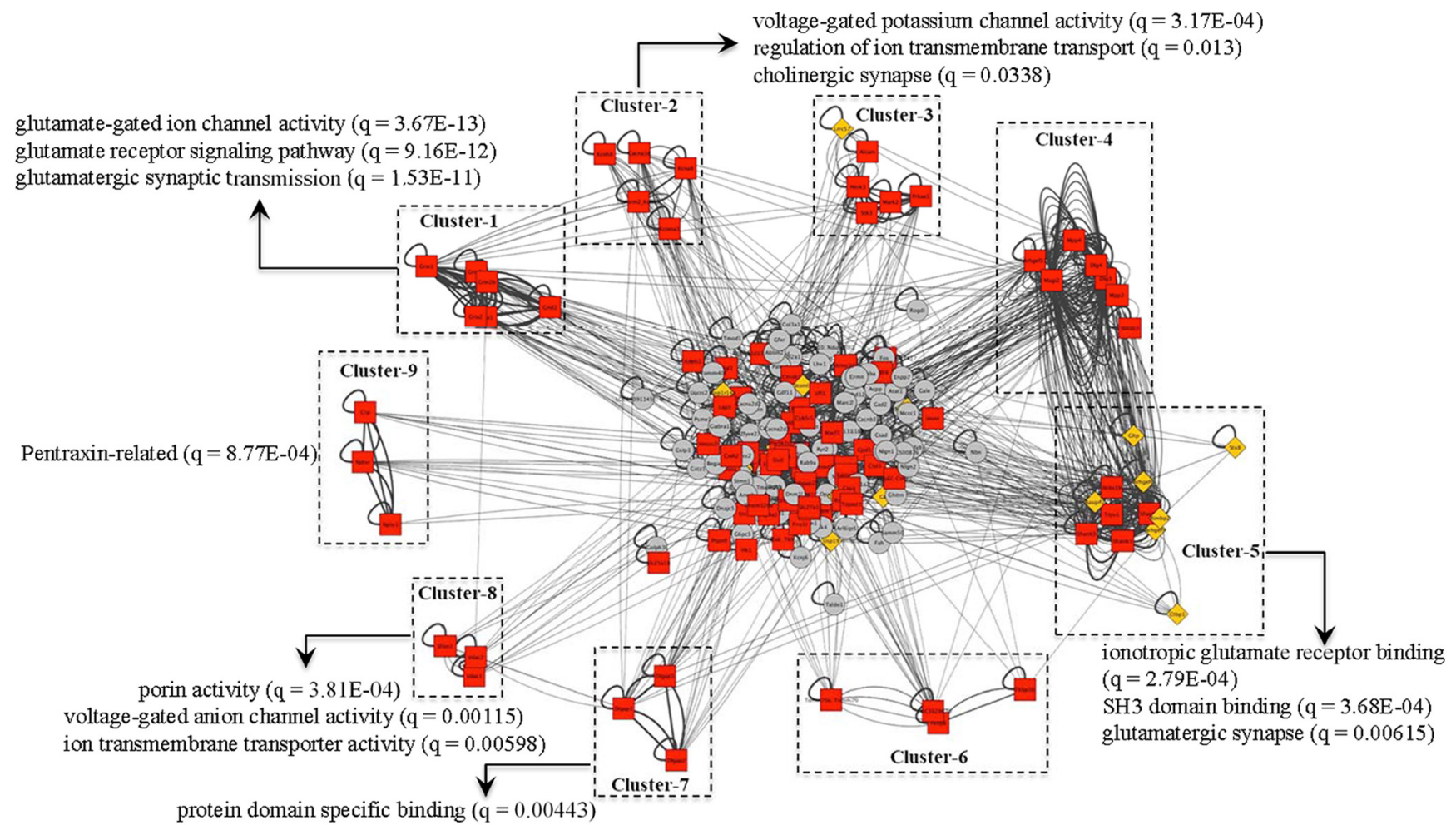

Figure 5. PSS network analysis. The BLASTP suite (BLASTP 2.8.0+) was used to search proteins and determine their sequence similarity. PSS network was created by using Cytoscape. The PSS network was clustered by using ClusterONE, and nine clusters were significantly identified. The edges were weighted by their sequence similarity and the enrichment analysis was performed for each of the nine clusters to identify specific signaling pathways of the clusters.

were clustered based on their sequence similarity. Among the 25 clusters identified, only nine clusters were statistically significant ( $p$ values for all clusters $<0.05$; Fig. 5 ). However, clusters 3,4 , and 6 did not reach statistical significance for the enrichment analysis (PPI enrichment $p$ $>0.05$ ). As shown in Figure 5, each cluster is significantly related to a specific functional enrichment annotation. The clusters 1 and 5 are significantly related to glutamatergic pathways while clusters 2 and 8 are associated with voltage-gated channel activities. Functional annotations of protein domain specific binding and Pentraxin were significantly associated with the clusters 7 and 9, respectively. Based on the results of subgraph centrality measure for the PSS network, the top 10 ranked proteins are MACF1, SHANK1, ARHGEF6, DLG3, MPP2, MPP4, ARHGEF9, SHANK3, SHANK2, SHANK2 isoform 4 and MAGI2. The expression of the nine proteins (SHANK2 isoform 4 excluded) was significantly different between the acquisition and sham groups $(p<0.05)$.

\section{Discussion}

In this study, we examined whether anodal tDCS could improve performance in a learning and memory task and the underlying protein modifications in the hippocampus, a brain region important for memory acquisition and recall. We have demonstrated that the timing of anodal tDCS is critical for memory performance. Anodal tDCS only improved memory performance when brain stimulation was applied before the acquisition period of the PAT. We further investigated the regulatory mechanisms for the memory enhancing effects of tDCS. Our results indicate tDCS induces changes in hippocampal synaptoneurosomes enhancing pathways related to cognition, learning, and memory. Additional analysis identified that the enhanced pathways are associated with specific receptor signaling and ion channel activity.

Our finding that anodal tDCS enhances cognitive performance when administered before memory acquisition is consistent with previous studies. A human-based study reported that tDCS enhances verbal memorization when anodal stimulation is applied during the memory encoding period (Javadi and Walsh, 2012). Another recent study shows that anodal tDCS delivered during the memory encoding period, but not the retrieval period, enhances memory (Medvedeva et al., 2019). Fertonani et al. (2014) also found that the timing of stimulation in relation to a cognitive task significantly affected performance. Together, previous studies and our current work suggest that tDCS-induced memory enhancement is dependent on the timing of tDCS administration.

To understand the underlying mechanisms for anodal tDCS-induced memory enhancement, we profiled proteins in the hippocampal synaptoneurosomes and analyzed the proteomic data. Compared to the sham treatment group, there were significant differences in protein abundances for the acquisition group (184 proteins). The PCA and hierarchical clustering analysis show a clear separation in distribution for the sham and acquisition groups. Enrichment and pathway analyses for the statis- 
tically significant proteins between the sham and acquisition group show changes in hippocampal protein regulation associated with synaptic neurotransmission and transporters. Using multiple database analyses from PantherDB and David bioinformatics (Tables 1 and 3), we further identified pathways involving glutamate receptor and ion channel signaling. Glutamate receptors and ion channels regulate neuronal activity and synaptic plasticity, which underlie neurologic processes of learning and memory (Gasque et al., 2006; Voglis and Tavernarakis, 2006; Gecz, 2010; Queenan et al., 2017; Foster et al., 2018). Although the underlying mechanisms of tDCS on learning and memory remain under investigation, our data show that anodal tDCS applied before the memory acquisition period modifies the regulation of hippocampal glutamate signaling and ion channel activity ultimately resulting in enhanced memory performance in rats.

We used IPA to understand hippocampal biological functions associated with learning and memory. Similar to the enrichment and pathway analysis results, the comparison between the acquisition and sham groups identified significant enhancements of memory, cognition, and learning while negative behaviors, such as anxiety, were significantly reduced. Our data show that anodal tDCS applied before the acquisition period modifies synaptic proteomic regulation in a manner associated with multiple electrophysiological and molecular pathways such as neuritogenesis, LTP, branching of neurites and synaptic transmission, which consequently enhance learning and memory. There is evidence that these pathways possibly explain the beneficial effects of tDCS on cognition. For example, tDCS has been reported to modulate LTP, which is well known to enhance cognition (Ranieri et al., 2012). Additionally, the networks developed by IPA contain many molecules that have been known to be associated with learning and memory. APOE, BDNF, GSK3B, and SHANK3 were identified as candidate regulators of the network to control memory and synaptic plasticity. PSEN1, MAPT1, APP, and L-dopa were identified as upstream regulators for significant proteins detected from the comparison between sham and acquisition groups. Most of these candidate regulators have been reported to be associated with cognition: APOE (Risacher et al., 2015; Sinclair et al., 2015), BDNF (Poo, 2001), PSEN1 (Kennard and Harrison, 2014), APP (Tamayev and D'Adamio, 2012), and L-DOPA (Murer and Moratalla, 2011). A recent study shows that tDCS induces BDNF expression and consequently enhances memory (Cocco et al., 2018). Based on the results of the Ingenuity upstream regulator analysis in IPA, our study is the first to suggest the following potential candidate regulators for anodal tDCS effects on learning and memory: GSK3b, SHANK3, and MAPT1. Most research on the role SHANK3 in the brain is related to autism (Yi et al., 2016). Recently, Duffney et al. (2013) showed that SHANK3 is associated with NMDA receptor function, suggesting a potential role for SHANK3 in learning and memory. Currently, there are no published reports linking GSK3b with tDCS-induced learning and memory, but a recent study reported that GSK3b is associated with Alzheimer's disease (Jayapalan and Natarajan, 2013). We cannot find any research that investigates roles of MAPT1 in learning and memory, but there is evidence that a MAPT mutation may be indirectly associated with cognitive dysfunction observed in dementia (lyer et al., 2013). More work is needed to elucidate the roles of these novel candidate molecules in learning and memory.

To identify significant protein networks for tDCSinduced effects on proteomic modifications of the hippocampal synapse, we conducted network analysis. The PPI network for the acquisition group compared to sham group is significantly associated with learning, memory, cognition, long-term memory, and glutamate receptor signaling pathways. Significant glutamate signaling pathways and ion channel activity were also identified from the PSS network. A relationship between ion channel activity and memory performance has been previously reported (Cavallaro, 2004) and it showed higher ion channel activity in animals is significantly associated with better memory performance as measured by the PAT. Glutamate receptor signaling pathways are also known to be dynamically regulated during learning and memory (Tronson and Taylor, 2007; Poo et al., 2016; Foster et al., 2018). Our studies have identified significant glutamate signaling and channel activity for the acquisition and sham group comparison using multiple analyses including enrichment and pathway analyses, IPA and PPI and PSS networks providing additional evidence for the mechanism of tDCS effects on hippocampal synaptoneurosomes. Therefore, our data suggest that anodal tDCS applied before the acquisition period modifies glutamate signaling and ion channel activity and consequently enhances memory performance. Our results identifying glutamatergic signaling as a mechanism for tDCS-induced effects on learning are supported by previous reports (Rossini et al., 2015; Giordano et al., 2017; Stafford et al., 2018). Our work is the first to use proteomics to suggest possible molecular effects associated with tDCS-enhancing memory acquisition by modifying glutamate signaling and ion channel activity pathways in rat hippocampal synaptoneurosomes.

From the PPI and PSS networks, we identified key molecules that lethally affect the protein networks. Key molecules in the networks identified by subgraph centrality measure include SHANK, DLG, GRIN, and GRIA proteins. Although future studies are needed to investigate the effects of these key players in the network, our work supports possible roles of these proteins in learning and memory. The function of glutamate receptor (Grin2a and Gria1) in learning and memory is well understood. Additionally, recent studies suggest SHANK may mediate the activity of glutamate receptors in neurons (Bertaso et al., 2010; Ha et al., 2018) and may play a role in synapse formation and plasticity (Kilinc, 2018). SHANK was also identified as one of the regulators in the network from the IPA suggesting that anodal tDCS modulates SHANK and subsequently affects synaptic plasticity and transmission in hippocampal neurons. Additionally, SHANK and DLG proteins were also identified as critical molecules in the PSS network. 
Bioinformatics and network analyses were completed for the comparisons of the retrieval group to the sham and acquisition group even through the behavioral data were not significant. Compared to the sham treatment group, there were significant differences in protein abundance for the retrieval group (82 proteins) Furthermore, the candidate regulators suggested from the Ingenuity upstream regulator analysis in IPA for the comparison between the sham and acquisition group (see above) did not result in a statistically significant difference. Additionally, the clustering analyses did not show a clear separation between the retrieval group and the sham and acquisition groups. Comparison of the acquisition group to the retrieval group using enrichment and pathway analyses, generated significant effects of hippocampal protein regulation of glutamate receptor pathways and ion channel pathways. Unique pathways identified for the acquisition and retrieval group comparison include pyrophosphatase activity and SNAP receptor activity. Many similar pathways were identified from the acquisition and sham versus the acquisition and retrieval group comparisons (Tables 1, 2), suggesting similarity between the sham and retrieval groups. More time poststimulation may be needed for the molecular modifications in the retrieval group to show a clear separation from the sham group.

In summary, we investigated whether anodal tDCS could affect memory acquisition, recall, or both, and the underlying molecular modifications in hippocampal synaptoneurosomes. We found that anodal tDCS administered before acquisition significantly enhanced memory performance potentially by enhancing the abundance of proteins associated with glutamate signaling and ion channel activity in hippocampal synapses. Our results also suggest potential molecular targets for the effects of anodal tDCS on memory performance. Our work contributes to the understanding of the regulatory molecular mechanisms of tDCS-induced memory enhancements. However, because the focus of our study is to observe molecular differences in hippocampal synapses and correlate proteomic changes with rodent cognitive performance, we should point out that our data do not report any cause-and-effect relationship between proteomic modifications, tDCS, and cognitive performance. Thus, future studies are needed to investigate the causal effects of the proteins identified from our study. Increased understanding of the mechanisms for the effects of tDCS on memory enhancement will facilitate future applications of brain stimulation.

\section{References}

Alonzo A, Brassil J, Taylor JL, Martin D, Loo CK (2012) Daily transcranial direct current stimulation (tDCS) leads to greater increases in cortical excitability than second daily transcranial direct current stimulation. Brain Stimul 5:208-213.

Ashtiani M, Salehzadeh-Yazdi A, Razaghi-Moghadam Z, Hennig H, Wolkenhauer O, Mirzaie M, Jafari M (2018) A systematic survey of centrality measures for protein-protein interaction networks. BMC Syst Biol 12:80.

Bailey NW, Thomson RH, Hoy KE, Hernandez-Pavon JC, Fitzgerald PB (2016) TDCS increases cortical excitability: direct evidence from TMS-EEG. Cortex 74:320-322.
Bertaso F, Roussignol G, Worley P, Bockaert J, Fagni L, Ango F (2010) Homer1a-dependent crosstalk between NMDA and metabotropic glutamate receptors in mouse neurons. PLoS One 5:e9755.

Bogdanov M, Schwabe L (2016) Transcranial stimulation of the dorsolateral prefrontal cortex prevents stress-induced working memory deficits. J Neurosci 36:1429-1437.

Boggio PS, Ferrucci R, Rigonatti SP, Covre P, Nitsche M, PascualLeone A, Fregni $F$ (2006) Effects of transcranial direct current stimulation on working memory in patients with Parkinson's disease. J Neurol Sci 249:31-38.

Cattaneo Z, Pisoni A, Papagno C (2011) Transcranial direct current stimulation over Broca's region improves phonemic and semantic fluency in healthy individuals. Neuroscience 183:64-70.

Cavallaro S (2004) DNA microarrays and animal models of learning and memory. Int Rev Neurobiol 60:97-133.

Cocco S, Podda MV, Grassi C (2018) Role of BDNF signaling in memory enhancement induced by transcranial direct current stimulation. Front Neurosci 12:427.

Duffney LJ, Wei J, Cheng J, Liu W, Smith KR, Kittler JT, Yan Z (2013) Shank3 deficiency induces NMDA receptor hypofunction via an actin-dependent mechanism. J Neurosci 33:15767-15778.

Fertonani A, Brambilla M, Cotelli M, Miniussi C (2014) The timing of cognitive plasticity in physiological aging: a tDCS study of naming. Front Aging Neurosci 6:131.

Flöel A, Suttorp W, Kohl O, Kürten J, Lohmann H, Breitenstein C, Knecht S (2012) Non-invasive brain stimulation improves objectlocation learning in the elderly. Neurobiol Aging 33:1682-1689.

Foster WJ, Taylor HBC, Padamsey Z, Jeans AF, Galione A, Emptage NJ (2018) Hippocampal mGluR1-dependent long-term potentiation requires NAADP-mediated acidic store $\mathrm{Ca}(2+)$ signaling. Sci Signal 11:eaat9093.

Gasque G, Labarca P, Delgado R, Darszon A (2006) Bridging behavior and physiology: ion-channel perspective on mushroom bodydependent olfactory learning and memory in Drosophila. J Cell Physiol 209:1046-1053.

Gecz J (2010) Glutamate receptors and learning and memory. Nat Genet 42:925-926.

Giordano J, Bikson M, Kappenman ES, Clark VP, Coslett HB, Hamblin MR, Hamilton R, Jankord R, Kozumbo WJ, McKinley RA, Nitsche MA, Reilly JP, Richardson J, Wurzman R, Calabrese E (2017) Mechanisms and effects of transcranial direct current stimulation. Dose Response 15:1559325816685467.

Göder R, Baier PC, Beith B, Baecker C, Seeck-Hirschner M, Junghanns K, Marshall L (2013) Effects of transcranial direct current stimulation during sleep on memory performance in patients with schizophrenia. Schizophr Res 144:153-154.

Ha HTT, Leal-Ortiz S, Lalwani K, Kiyonaka S, Hamachi I, Mysore SP, Montgomery JM, Garner CC, Huguenard JR, Kim SA (2018) Shank and zinc mediate an AMPA receptor subunit switch in developing neurons. Front Mol Neurosci 11:405.

Hill AT, Rogasch NC, Fitzgerald PB, Hoy KE (2019) Impact of concurrent task performance on transcranial direct current stimulation (tDCS)-Induced changes in cortical physiology and working memory. Cortex 113:37-57.

Holland R, Leff AP, Josephs O, Galea JM, Desikan M, Price CJ, Rothwell JC, Crinion J (2011) Speech facilitation by left inferior frontal cortex stimulation. Curr Biol 21:1403-1407.

Holmes B, Jung SH, Lu J, Wagner JA, Rubbi L, Pellegrini M, Jankord $R$ (2016) Transcriptomic modification in the cerebral cortex following noninvasive brain stimulation: RNA-sequencing approach. Neural Plast 2016:5942980.

Hoy KE, Emonson MR, Arnold SL, Thomson RH, Daskalakis ZJ, Fitzgerald PB (2013) Testing the limits: investigating the effect of tDCS dose on working memory enhancement in healthy controls. Neuropsychologia 51:1777-1784.

Huang da W, Sherman BT, Lempicki RA (2009) Bioinformatics enrichment tools: paths toward the comprehensive functional analysis of large gene lists. Nucleic Acids Res 37:1-13. 
Iyer A, Lapointe NE, Zielke K, Berdynski M, Guzman E, Barczak A, Chodakowska-Z'ebrowska M, Barcikowska M, Feinstein S, Zekanowski C (2013) A novel MAPT mutation, G55R, in a frontotemporal dementia patient leads to altered Tau function. PLoS One 8:e76409.

Jacobson L, Goren N, Lavidor M, Levy DA (2012) Oppositional transcranial direct current stimulation (tDCS) of parietal substrates of attention during encoding modulates episodic memory. Brain Res 1439:66-72.

Javadi AH, Walsh V (2012) Transcranial direct current stimulation (tDCS) of the left dorsolateral prefrontal cortex modulates declarative memory. Brain Stimul 5:231-241.

Javadi AH, Cheng P (2013) Transcranial direct current stimulation (tDCS) enhances reconsolidation of long-term memory. Brain Stimul 6:668-674.

Jayapalan S, Natarajan J (2013) The role of CDK5 and GSK3B kinases in hyperphosphorylation of microtubule associated protein tau (MAPT) in Alzheimer's disease. Bioinformation 9:1023-1030.

Jeong H, Mason SP, Barabási AL, Oltvai ZN (2001) Lethality and centrality in protein networks. Nature 411:41-42.

Kennard JA, Harrison FE (2014) Intravenous ascorbate improves spatial memory in middle-aged APP/PSEN1 and wild type mice. Behav Brain Res 264:34-42.

Kilinc D (2018) The emerging role of mechanics in synapse formation and plasticity. Front Cell Neurosci 12:483.

Kim MS, Koo H, Han SW, Paulus W, Nitsche MA, Kim YH, Yoon JA, Shin YI (2017) Repeated anodal transcranial direct current stimulation induces neural plasticity-associated gene expression in the rat cortex and hippocampus. Restor Neurol Neurosci 35:137-146.

Ladenbauer J, Ladenbauer J, Külzow N, de Boor R, Avramova E, Grittner U, Flöel A (2017) Promoting sleep oscillations and their functional coupling by transcranial stimulation enhances memory consolidation in mild cognitive impairment. J Neurosci 37:71117124.

Marshall L, Mölle M, Hallschmid M, Born J (2004) Transcranial direct current stimulation during sleep improves declarative memory. $\mathrm{J}$ Neurosci 24:9985-9992.

Martin DM, Liu R, Alonzo A, Green M, Loo CK (2014) Use of transcranial direct current stimulation (tDCS) to enhance cognitive training: effect of timing of stimulation. Exp Brain Res 232:3345-3351.

Medvedeva A, Materassi M, Neacsu V, Beresford-Webb J, Hussin A, Khan N, Newton F, Galli G (2019) Effects of anodal transcranial direct current stimulation over the ventrolateral prefrontal cortex on episodic memory formation and retrieval. Cereb Cortex 29:657665.

Mi H, Muruganujan A, Thomas PD (2013) PANTHER in 2013: modeling the evolution of gene function, and other gene attributes, in the context of phylogenetic trees. Nucleic Acids Res 41:D377D386.

Montojo J, Zuberi K, Rodriguez H, Kazi F, Wright G, Donaldson SL, Morris Q, Bader GD (2010) GeneMANIA cytoscape plugin: fast gene function predictions on the desktop. Bioinformatics 26:29272928.

Murer MG, Moratalla R (2011) Striatal signaling in L-DOPA-induced dyskinesia: common mechanisms with drug abuse and long term memory involving D1 dopamine receptor stimulation. Front Neuroanat 5:51.

Nepusz T, Yu H, Paccanaro A (2012) Detecting overlapping protein complexes in protein-protein interaction networks. Nat Methods 9:471-472.

Podda MV, Cocco S, Mastrodonato A, Fusco S, Leone L, Barbati SA, Colussi C, Ripoli C, Grassi C (2016) Anodal transcranial direct current stimulation boosts synaptic plasticity and memory in mice via epigenetic regulation of Bdnf expression. Sci Rep 6:22180.

Poo MM (2001) Neurotrophins as synaptic modulators. Nat Rev Neurosci 2:24-32.

Poo MM, Pignatelli M, Ryan TJ, Tonegawa S, Bonhoeffer T, Martin KC, Rudenko A, Tsai LH, Tsien RW, Fishell G, Mullins C, Gonçalves JT, Shtrahman M, Johnston ST, Gage FH, Dan Y, Long J,
Buzsáki G, Stevens C (2016) What is memory? The present state of the engram. BMC Biol 14:40.

Queenan BN, Ryan TJ, Gazzaniga MS, Gallistel CR (2017) On the research of time past: the hunt for the substrate of memory. Ann NY Acad Sci 1396:108-125.

Ranieri F, Podda MV, Riccardi E, Frisullo G, Dileone M, Profice P, Pilato F, Di Lazzaro V, Grassi C (2012) Modulation of LTP at rat hippocampal CA3-CA1 synapses by direct current stimulation. $J$ Neurophysiol 107:1868-1880.

Risacher SL, Kim S, Nho K, Foroud T, Shen L, Petersen RC, Jack CR Jr, Beckett LA, Aisen PS, Koeppe RA, Jagust WJ, Shaw LM, Trojanowski JQ, Weiner MW, Saykin AJ; Alzheimer's Disease Neuroimaging Initiative (ADNI) (2015) APOE effect on Alzheimer's disease biomarkers in older adults with significant memory concern. Alzheimers Dement 11:1417-1429.

Rohan JG, Carhuatanta KA, McInturf SM, Miklasevich MK, Jankord R (2015) Modulating hippocampal plasticity with in vivo brain stimulation. J Neurosci 35:12824-12832.

Romero Lauro LJ, Rosanova M, Mattavelli G, Convento S, Pisoni A, Opitz A, Bolognini N, Vallar G (2014) TDCS increases cortical excitability: direct evidence from TMS-EEG. Cortex 58:99-111.

Rossini PM, Burke D, Chen R, Cohen LG, Daskalakis Z, Di lorio R, Di Lazzaro V, Ferreri F, Fitzgerald PB, George MS, Hallett M, Lefaucheur JP, Langguth B, Matsumoto $\mathrm{H}$, Miniussi $\mathrm{C}$, Nitsche MA, Pascual-Leone A, Paulus W, Rossi S, Rothwell JC, et al. (2015) Non-invasive electrical and magnetic stimulation of the brain, spinal cord, roots and peripheral nerves: basic principles and procedures for routine clinical and research application. An updated report from an I.F.C.N. Committee. Clin Neurophysiol 126:10711107.

Shannon P, Markiel A, Ozier O, Baliga NS, Wang JT, Ramage D, Amin N, Schwikowski B, Ideker T (2003) Cytoscape: a software environment for integrated models of biomolecular interaction networks. Genome Res 13:2498-2504.

Sinclair LI, Button KS, Munafò MR, Day IN, Lewis G (2015) Possible association of APOE genotype with working memory in young adults. PLoS One 10:e0135894.

Stafford J, Brownlow ML, Qualley A, Jankord R (2018) AMPA receptor translocation and phosphorylation are induced by transcranial direct current stimulation in rats. Neurobiol Learn Mem 150:36-41.

Stagg CJ, Nitsche MA (2011) Physiological basis of transcranial direct current stimulation. Neuroscientist 17:37-53.

Szklarczyk D, Morris JH, Cook H, Kuhn M, Wyder S, Simonovic M, Santos A, Doncheva NT, Roth A, Bork P, Jensen LJ, von Mering C (2017) The STRING database in 2017: quality-controlled proteinprotein association networks, made broadly accessible. Nucleic Acids Res 45:D362-D368.

Tamayev R, D'Adamio L (2012) Memory deficits of British dementia knock-in mice are prevented by $A \beta$-precursor protein haploinsufficiency. J Neurosci 32:5481-5485.

Tronson NC, Taylor JR (2007) Molecular mechanisms of memory reconsolidation. Nat Rev Neurosci 8:262-275.

Voglis G, Tavernarakis N (2006) The role of synaptic ion channels in synaptic plasticity. EMBO Rep 7:1104-1110.

Wang J, Li M, Wang H, Pan Y (2012) Identification of essential proteins based on edge clustering coefficient. IEEE/ACM Trans Comput Biol Bioinform 9:1070-1080.

Warde-Farley D, Donaldson SL, Comes O, Zuberi K, Badrawi R, Chao P, Franz M, Grouios C, Kazi F, Lopes CT, Maitland A, Mostafavi S, Montojo J, Shao Q, Wright G, Bader GD, Morris Q (2010) The GeneMANIA prediction server: biological network integration for gene prioritization and predicting gene function. Nucleic Acids Res 38:W214-W220.

Yi F, Danko T, Botelho SC, Patzke C, Pak C, Wernig M, Südhof TC (2016) Autism-associated SHANK3 haploinsufficiency causes Ih channelopathy in human neurons. Science 352:aaf2669.

Yu TH, Wu YJ, Chien ME, Hsu KS (2019) Transcranial direct current stimulation induces hippocampal metaplasticity mediated by brain-derived neurotrophic factor. Neuropharmacology 144:358367. 